\title{
NASOGASTRIC TUBE PLACEMENT- A SIMPLE YET DIFFICULT PROCEDURE- A REVIEW
}

\author{
Mohanchandra Mandal1, Dipanjan Bagchi², Susanta Sarkar3, Piyali Chakrabarti ${ }^{4}$, Suchitra Pal ${ }^{5}$ \\ ${ }^{1}$ Associate Professor, Department of Anaesthesiology, North Bengal Medical College, Sushrutanagar, Darjeeling, West Bengal. \\ ${ }^{2}$ Consultant Anaesthesiologist, Howrah District Hospital, Howrah, West Bengal. \\ ${ }^{3}$ Associate Professor, Department of Anaesthesiology, North Bengal Medical College, Sushrutanagar, Darjeeling, West Bengal. \\ ${ }^{4}$ Postgraduate Trainee, Department of Physiology, North Bengal Medical College, Sushrutanagar, Darjeeling, West Bengal. \\ ${ }^{5}$ Nursing Sister, M. Sc. Nursing (Psychiatry), Central Hospital, SE Railway Garden Reach, Kolkata, West Bengal.
}

\section{ABSTRACT}

\section{BACKGROUND}

Summary- Despite several novel techniques reported in the literature regarding nasogastric tube placement, no technique has emerged as the most efficient method, especially for unconscious patients. A few of them appears to be achieving higher success rate and considered to be better than the rest. Varied complications have been reported in the literature. We searched for relevant medical literature in English using Google Search Engine. The following Medical Subject Headings (MeSH) terms were used: Decompression, Intubation, Gastrointestinal; Intubation, Intratracheal, Parenteral Nutrition, Stomach. The search was further extended using related keywords such as 'nasogastric intubation,' 'nasogastric tube insertion,' 'nasogastric tube intubation,' 'nasogastric tube placement.' The full text articles published in 2000 onwards were mainly considered with the exception for some old seminal articles. Primarily, original investigations, editorials, letter to Editor and brief communications were consulted. A few review articles were also taken into consideration. A brief outline about nasogastric tubes, its application, confirmatory tests, their current status with loopholes, different method to increase the objectivity of successful placement of nasogastric tube, etc. have been described. Mostly, a general view regarding this has been presented with a mention about the gray zones.

\section{KEYWORDS}

Gastric Tube, Nasogastric Tube, Nasogastric Tube Placement, Orogastric Tube, Intubation.

HOW TO CITE THIS ARTICLE: Mandal M, Bagchi D, Sarkar S, et al. Nasogastric tube placement- a simple yet difficult procedure- a review. J. Evolution Med. Dent. Sci. 2017;6(31):2572-2576, DOI: 10.14260/Jemds/2017/556

\section{BACKGROUND}

Successful placement of Nasogastric Tube (NGT) in anaesthetised or unconscious individuals in operating room and intensive care unit appears to be challenging one compared with its insertion in conscious patients. It is introduced through the nose into the stomach, mainly for providing short-term or medium-term supplementary nutrition for giving gastric lavage or for decompressing the stomach- the last purpose being most important in anaesthetised patients during surgery. It is frequently needed in patients undergoing thoracic and abdominal surgeries. Its insertion in the unconscious and paralysed patients is often not so easy. Convention method of placement of NGT bears a failure rate of around $50 \% .1,2$ In this narrative review, an endeavour has been made to depict the general outline about NGT, different novel techniques, their success rates and comparative status and various complications during its placement. Methods for confirmation of successful placement of NGT and techniques to increase the objectivity and safety of blind insertion of NGT have been described.

\section{Types of Nasogastric Tubes}

The Nasogastric Tube (NGT) is a narrow tube, made of Polyvinyl Chloride (PVC) or Polyurethane (PUC). The latter is more flexible and less prone to cause trauma. 3,4

Financial or Other, Competing Interest: None.

Submission 04-03-2017, Peer Review 05-04-2017,

Acceptance 12-04-2017, Published 17-04-2017.

Corresponding Author:

Mohanchandra Mandal,

A-5, M. O. Quarters, N. B. M. C. Campus,

P. O. Sushrutanagar, Dist-Darjeeling-734012,

West Bengal, India.

E-mail: drmcmandal@gmail.com

DOI: $10.14260 /$ jemds $/ 2017 / 556$
The weighted nasogastric tubes have tungsten ball instead of mercury ones, reducing the risk of inadvertent absorption and toxicity. The tip of the weighted tube points preferentially towards the posterior oropharynx and as such has a greater chance to enter the oesophagus. ${ }^{3,4}$ Many NGTs used today are flexible and need guidewires or stilettes for their insertion. They may be large bore $(14 \mathrm{~F})$ or small bore (8-12F). The larger ones are made of the tougher PVC and have lesser chance of malposition or kinking and have greater reliability for aspiration of gastric contents and confirmation of position. They can be introduced at the bedside by a trained nurse. ${ }^{4}$ Again NGTs may be classified as 'passive' or 'active' depending upon how it functions. The passive could be 'open' or 'closed.' The NGT is left open or connected to a collecting system (closed) without the application of suction machine. NGT is termed as 'active' when it is connected to a suction machine to produce a slow but consistent evacuation of the gastric material. Intermittent connection to suction machine achieves better result than a continuous one. ${ }^{5}$

\section{Feeding by Nasogastric Tubes 6}

- Bolus feed is simple, requiring minimal equipment, executed with the help of gravity. However, it might increase the risk of gastrointestinal (GI) symptoms.

- Intermittent feed using the help of gravity or pump. It allows some feeding-free interval, but increases the propensity of GI symptoms.

- Continuous feed using a pump system: this reduces the incidence of GI symptoms, but the patient remains connected to the system most of the time and hence limit mobility.

- $\quad$ Semi-recumbent positioning reduces the propensity of airway aspiration. 
- Contamination of feeds can be curtailed by minimal and careful handling and the use of closed systems rather than open one.

\section{Contraindications of NGT Insertion}

Feeding through NGT is not suitable in some clinical scenario, such as substantial maxillofacial trauma, recent history of ingestion of caustic materials, oesophageal strictures and diverticula, severe nasal injuries, base of skull fractures, etc. In patients with altered mental status or impaired airway defenses, NGT insertion should not be tried before securing airway with endotracheal intubation as the former may precipitate vomiting and aspiration. 6

\section{Preparing the Patients for Nasogastric Tube Placement} Frequently, a lubricating jelly ( $2 \%$ lignocaine, $\mathrm{K}-\mathrm{Y}$ jelly or any non-anaesthetic lubricant jelly) and a nasal decongestant is used to aid the insertion of the NGT. Current recommendations include $2 \%$ lignocaine jelly, nebulisation with $4 \%$ lignocaine, atomised $4 \%$ lignocaine nasal spray, $4 \%$ lignocaine and oxymetazoline atomised nasal spray and oxymetazoline nasal spray.7,8 In the anaesthetised patient, $\mathrm{K}$ $\mathrm{Y}$ jelly can be easily used. $\mathrm{K}-\mathrm{Y}$ jelly is a simple lubricating jelly which contains glycerine and hydroxymethyl cellulose as lubricating material. Chlorhexidine gluconate and gluconodelta-lactone act as antiseptics. It also contains methylparaben and sodium hydroxide as preservative agents.

\section{Confirmation of Position \\ Clinical}

The confirmation of position of a nasogastric tube can be done clinically by- i) Examining the length of the tube introduced beyond the nostril, ii) Ausculting over the epigastrium while deflating an air-filled syringe attached to the proximal end of the tube, iii) Aspirating the gastric contents through the NGT. Combination of all three yields the best result. Of these, auscultation is the commonest and most popular. But, loopholes exist. A gurgling sound can be heard over the epigastrium even if the NGT is in the tracheabronchial tree, pleural space or oesophagus. ${ }^{9}$

\section{Testing pH of Aspirate}

Gastric placement is indicated by a $\mathrm{pH}$ of less than 4 , but may increase in the range of $4-6$ in case of acid-inhibiting drug therapy. The use of blue litmus paper to check the acidity of aspirate material lacks sensitivity to distinguish between levels of acidity.10,11 Magnetic guidance can rule out lung or oesophageal placement- the two most hazardous potential tube sites, whereas a pH test with cut-offs at 5.5 or lower can rule out lung misplacements. There is potential of magnetic guidance testing to be the safest option followed by the $\mathrm{pH}$ test with a cut-off of 4.0 or 5.5. However, the pH test remains the safest test when using a cut-off of 4.0.12

\section{X-Rays}

This will confirm position at only the time the x-ray is carried out. The tube may get displaced by the time when patient returns to ward. In the absence of $\mathrm{pH}$ testing facility, an x-ray must be repeated to confirm the initial position of the NGT.13 X-ray also ensures that the ports of an NGT are in the stomach and therefore it is properly positioned in the gastrointestinal tract. Even nasogastric tubes that ended in the distal oesophagus can result in aspiration. Many experts and professional bodies agree that the cost of an x-ray to confirm correct placement of a blindly inserted tube prior to its use to administer feeds or medications should be regarded as justified. 14

An NGT is considered 'malpositioned' if it is not within the oesophageal lumen or if the distal end of the tube is not below the gastro-oesophageal junction. To place an NGT, it is assumed that the median distance from the anterior nasal spine to the tracheo-oesophageal junction to be about $20 \mathrm{~cm}$, the oesophagus to be $25 \mathrm{~cm}$ long and to aim for the tip of the NGT to lie $10 \mathrm{~cm}$ below the gastro-oesophageal junction. Therefore, ideally the NGT should be secured at the 50 to 60 $\mathrm{cm}$ mark at the nasal vestibule. Alternatively, the length of insertion is determined by adding the distances from nose to pinna and the distance from pinna to the xiphoid process plus extra $5 \mathrm{~cm}$ allowance. This will place the tip of the NGT in the fundus of the stomach. ${ }^{14}$

\section{When and how many times should the tube position be checked?}

\section{The Position of NGT should be checked in the following} Manners-

- It should be checked after initial placement and thereafter at least once daily during continuous feeds. It is essential to check tube position before the start of feed following a break.

- It should be checked in case of reflux of feed and after episodes of coughing, retching or vomiting after oropharyngeal suction or if there is any discomfort or respiratory distress.

- $\quad$ Before administration of any drug if NGT is not being used for any other purpose.

- Its position should be checked and noted during transport of patient or if any change in length of the visible part of tube is noticed.

\section{Why is it difficult to Introduce NGT in Anaesthetised Patient?}

The piriform sinuses and arytenoids cartilages have been incriminated as the most common sites of impaction. ${ }^{15}$ The distal portion of the NGT has multiple apertures and thus is the weakest part. This makes the NGT susceptible to kinking, coiling and knotting when it encounters some resistance in its journey through nasopharynx and laryngopharynx. After a failure, subsequent attempts using the same NGT and applying the same technique leads to the same outcome (kinking at the same place) resulting in low success rate owing to the 'memory effect.'16 Flegar and Ball17 described one technique utilising refrigeration of nasogastric tube, while in-situ within package to make it rigid with a 'memory' for its coiled shape. The nasogastric tube should be inserted through nose with the concave side 'hugging the floor' of the nasal cavity. After reaching the oropharynx, the tube is rotated 180 degrees to bring its tip up against the posterior pharyngeal wall. This will allow the tip to stay over the posterior pharyngeal wall, facilitating its entrance into oesophagus. At this time point it is important to lift the chin and observe the neck, whilst slowly feeding the nasogastric tube. Any bulging noticed at the neck can be rectified by slight rotation of the tube. The authors opined this technique as a 'simple, but very useful technique' which may prove 
invaluable both in operating room and in intensive care unit. ${ }^{17}$

\section{Techniques of NGT Placement: A Flood in the Literature}

To overcome the difficulties mentioned above, many people have adopted different techniques, e.g. 'head flexion' $(80 \%$ success rate encountered in the 'flexion group' compared to $50 \%$ in the 'neutral group') 1 , 'reverse Sellick's manoeuvre' or anterior lifting of the cricoid's cartilage with a success rate $75 \%$ to $80 \%,{ }^{18}$ and the 'slit-tracheal tube'-guided insertion ${ }^{19}$ where the nasogastric tube is put inside a longitudinally slit endotracheal tube, which is then inserted blindly into the oral cavity up to a length of $18 \mathrm{~cm}$ and then withdrawn leaving the NGT inside. In the last study, Appukutty et $\mathrm{al}^{19}$ found a success rate of $94 \%, 92 \%$ and $92 \%$ within two attempts using the 'neck flexion with lateral pressure,' 'ureteral guidewireassisted technique' and 'slit-tracheal tube-guided methods', respectively.

While using a 'Rusch' intubation stylet and attaching a slipknot to tie the NGT to its tip ('slipknot to intubation stylet'), the success rate was found to be around $98 \%{ }^{20}$ Frozen NGT technique ${ }^{21}$ by filling a silicone NGT with distilled water and freezing it, has been reported a success rate of around $88 \%$; and the use of oesophageal guidewire 22 has been claimed to have a higher success rate of around 99\%. Kumar $\mathrm{P}$ et $\mathrm{al}^{23}$ used gloved fingers to guide the NGT along the posterior pharyngeal wall, whereas Kayo R et $\mathrm{al}^{24}$ used a $5 \mathrm{~cm}$ pillow beneath the patient's head.

Sometimes, the NGT has been introduced under direct endoscopic visions. Use of the Glide Scope had a first-attempt insertion rate of NGT of around 85\%. ${ }^{25-27}$ The nasolaryngoscope was used by Boston $\mathrm{AG}$ et $\mathrm{al}^{28}$ for inserting the NGT under direct visualisation with minimal trauma with the distal tip of the NGT being manipulated through a suture connecting the distal ends of NGT and the endoscope; however, this process needed active swallowing by the patient and is questionable in anaesthetised ones. The King Vision video-laryngoscope was used orally for guiding the NGT introduced through the nose through the piriform sinus or the oesophagus. ${ }^{29}$ This has been reported to have $100 \%$ efficacy and minimal complications, but is not always available at hand. A Seldinger technique of gliding NGT over nasoendoscope has also been mentioned in the recent past. 30

A combination of four steps one after another, viz. sniffing position, nasogastric tube orientation, contralateral rotation and twisting movement (SORT) achieved a high success rate. $^{31}$

Myriad of other techniques have been employed to help in NGT insertion. Prior inflation of pharynx with a facemask and self-inflating bag via two positive pressure breaths of 500 $600 \mathrm{~mL}$ was claimed to open up the collapsed oesophagus in the anaesthetised patient, owing to which the NGT could be introduced with much ease (success rate 96\%). ${ }^{32}$ Dobson et al ${ }^{33}$ used an innovative 'peel-away tube technique' by using a perforated endotracheal tube split near the distal end, which could be easily peeled off after the NGT had been introduced into the oesophagus wrapped in the ET tube. Application of lateral neck pressure ${ }^{2}$ was claimed to have a success rate of $80 \%$ at the first attempt. There is report of NGT insertion with an angiography catheter placed inside the NGT to strengthen it, the ET tube cuff having been inflated so as to have an audible leak and the cricoid cartilage lifted outwards and rightward (Samanta and Ghatak's technique). ${ }^{34}$ The technique of 'neck flexion and lateral pressure' also has been claimed to achieve a high success rate within two attempts; $88 \%{ }^{16}$ and $94 \%{ }^{19}$ in two different studies, respectively. The frozen NGT technique 20 was found to achieve $88 \%$ success rate regarding proper placement of NGT. The reverse Sellick's manoeuvre have been reported to achieve success rates of $75 \%-80 \% 18$ and $96 \%{ }^{35}$ within two attempts in two different studies, respectively.

\section{Techniques tried to Increase the Objectivity and Safety of Blind Insertion of NGT}

In 1989, Roubenoff and Ravich ${ }^{36}$ proposed a 'two-step protocol' for the NGT insertion. Here, the tube is initially advanced blindly to $30 \mathrm{~cm}$ and the position is verified by an $\mathrm{x}$ ray. This initial check is crucial to prevent a pulmonary malposition by keeping an already misdirected tube away from the more distal smaller bronchi or the lung, where a perforation is most likely. At the same time, the $30 \mathrm{~cm}$ length allows it to reach only the proximal main stem bronchi, so that the abnormal curve of deviation away from the midline will be detected on the x-ray and the procedure is halted. If the $x$-ray shows a midline tube, this confirms position of NGT to be in the oesophagus and the NGT then can be further advanced to the optimum length of $50 \mathrm{~cm}$ and confirmed with a second $\mathrm{x}$-ray. The 2 -step insertion procedure eliminates the potential for complications at the cost of two radiologic exposures and more time; hence, is not routinely practised. ${ }^{3}$ Marderstein EL et $\mathrm{al}^{37}$ modified the Roubenoff and Ravich's 'two-step protocol' with application of a pause when the NGT reached $35 \mathrm{~cm}$. Marderstein and co-researchers ${ }^{37}$ reported that with this two-step approach, no tube placed in the oesophagus has caused pulmonary damage.

Thomas BW et al (1998) ${ }^{38}$ used a colorimetric end-tidal $\mathrm{CO}_{2}$ indicator device, which uses a sulphapthaleinimpregnated $\mathrm{pH}$-sensitive filter paper that changes from purple to yellow in the presence of $\mathrm{CO}_{2}$. The authors suggested that it was $100 \%$ sensitive and specific in discriminating between the tubes passed into the airways and those passed into the alimentary tract.

Haddad $\mathrm{N}$ et al (1993) ${ }^{39}$ used an external transmitter with an electromagnetic pulse sensor system at the bedside to observe the location of the tip of the tube, as it is manually inserted. Prima facie, the reports suggest about its high success rates and decreased insertion time. Bercik $\mathrm{P}$ et al ${ }^{40}$ monitored the tip of NGT using a magnet-tipped computer tracking system during its insertion and compared it with simultaneous monitoring with fluoroscopy and manometry. An excellent correlation was found between the three techniques. Sorokin R and Gottlieb JE41 applied three changes to reduce the possibility of pneumothorax from NGT insertion in intubated patients. First, the NGT were either not advanced beyond $35 \mathrm{~cm}$ until an x-ray was obtained or were advanced under direct laryngoscopic view, fluoroscopic guidance or capnometric monitoring guidance. Second, they monitored the NGT malposition and reported to the clinical staff regularly. Third, training (orientation) program on NGT insertion among residents was started. 


\section{The pH and Bilirubin Estimation of the Aspirate Position Status}

Mean pH levels of inner environment of lungs and intestine are significantly higher than that of the stomach $(7.73,7.35$ and 3.90, respectively). However, after acquiring infection, pleural or respiratory secretion can show an acidic $\mathrm{pH}$ and a false positive for the gastric position. Misleading alkaline $\mathrm{pH}$ in the stomach can be seen in case of achlorhydria and with the use of potent anti-acid drugs. Proper (gastric) placement is indicated by a pH below 4 . The $\mathrm{pH}$ may increase to $4-6$ in those receiving acid-inhibiting therapy. The blue litmus paper was found insufficiently sensitive to distinguish between the levels of acidity.3,10,11 Magnetic-guidance can rule out lung or oesophageal placement, both are potentially hazardous. In contrast, a pH test with cut-off point set at 5.5 or lower can rule out NGT misplacement in lungs. There is potential of magnetic-guidance testing to be the safest option, followed by the $\mathrm{pH}$ test with a cut-off set at 4.0 or 5.5. A higher cut-off point of $\mathrm{pH}$ should only be used when the clinicians have sufficient experience and the $\mathrm{pH}$ is measured accurately. Hence, the $\mathrm{pH}$ test may be the safest test when using a cut-off of 4.0.12

Mean bilirubin levels in the lung $(0.08 \mathrm{mg} / \mathrm{dL})$ and stomach $(1.28 \mathrm{mg} / \mathrm{dL})$ were considerably lower than in the intestine $(12.73 \mathrm{mg} / \mathrm{dL})$. Measurement of bilirubin is now possible using a colourimetric test-strip with visual scale. Metheny $\mathrm{NA}^{42}$ combined these 2 markers and proposed a predictive, useful yet simple, bedside test. (i) A pH less than 5 and bilirubin less than $5 \mathrm{mg} / \mathrm{dL}$ of NGT aspirate identified $98 \%$ of gastric sites. (ii) A pH greater than 5 and a bilirubin less than $5 \mathrm{mg} / \mathrm{dL}$ of NGT aspirate identified $100 \%$ of the respiratory sites. (iii) $\mathrm{A} \mathrm{pH}$ greater than 5 and bilirubin greater than $5 \mathrm{mg} / \mathrm{dL}$ of the NGT aspirate has identified nearly $88 \%$ of the intestinal sites. However, this method only confirms the complication and does not prevent it.

\section{Capnography}

The presence of carbon dioxide $\left(\mathrm{CO}_{2}\right)$ is a surrogate marker for inadvertent pulmonary placement of NGT. Incorporating capnography into Roubenoff and Ravich's '2-step protocol,'3,36 could potentially increase its practical utility and can avert one extra radiological exposure compared with its original protocol. Araujo $\mathrm{P}^{43}$ reported excellent initial results with a compact, disposable colourimetric end-tidal $\mathrm{CO}_{2}$ detector. It was noted that the capnometer confirms the tube position at the crucial $30 \mathrm{~cm}$ position. The capnometer was found to detect $\mathrm{CO}_{2}$ coming out of the NGT reliably even with the guidewire in-situ. The tube is further inserted to $50 \mathrm{~cm}$ with a single radiologic confirmation for the final placement. They reported this colourimetric end-tidal $\mathrm{CO}_{2}$ detector to have $100 \%$ specificity and a $100 \%$ sensitivity rate in confirming successful NGT placement. 43

\section{Complications of NGT Insertion}

The most common complication from insertion of NGT is the coiling in the pharynx or oesophagus. If the side holes are positioned within the oesophagus, there is every possibility of aspiration; hence, the tip of NGT should be at least $10 \mathrm{~cm}$ caudal to location of the gastro-oesophageal junction. Fine bore nasoenteric tube have been in use for over three decades. Reported overall complication rates vary widely from 0.3 to $8.0 \%$. Several thoracic (bronchial placement and intravascular penetration) and non-thoracic (Enteral and intracranial) complications have been reported.3,44

Bronchial placement can lead to various complications such as atelectasis, pneumonia and lung abscess, bronchial perforation, pulmonary laceration, pulmonary haemorrhage, pleural cavity penetration and their consequences such as pneumothorax, empyema, pleural knotted tube, etc. The inadvertent insertion into the trachea or bronchial tree occurs in approximately $0.2 \%-0.3 \%$ of patients. Rarely, pharyngeal and oesophageal perforations can occur with serious consequences.3,14,44,45 Intravascular penetration occurred after erosion into retro-oesophageal aberrant right subclavian artery, erosion into right internal jugular vein and then to right atrium, etc. Enteral complications such as tube knotting and impaction in the posterior nasopharynx, tube breakage after being brittle with time, oesophageal perforation leading to mediastinitis and duodenal perforation- all have been reported. Intracranial entry has been observed following repair of choanal atresia and transnasal trans-sphenoidal surgery and in maxillofacial trauma patients.

To summarise, the procedure of nasogastric tube insertion may seem to be an easy one in daily practice. But, when the situation demands that one be inserted during an operation in an anaesthetised patient, it may not be that simple. Many have tried out different methods to ease out the process and many will in the future. Which procedure is the best depends on the expertise of the anaesthesiologist and the situation in hand, and should better not be generalised.

\section{REFERENCES}

[1] Mahajan R, Gupta R. Another method to assist nasogastric tube insertion. Can J Anaesth 2005;52(6):652-3.

[2] Bong CL, Macachor JD, Hwang NC. Insertion of the nasogastric tube made easy. Anesthesiology 2004;101(1):266.

[3] Pillai JB, Vegas A, Brister S. Thoracic complications of nasogastric tube: review of safe practice. Interact Cardiovasc Thorac Surg 2005;4(5):429-33.

[4] Cresci G, Mellinger J. The history of nonsurgical enteral tube feeding access. Nutr Clin Pract 2006;21(5):522-8.

[5] Makama JG. Uses and hazards of nasogastric tube in gastrointestinal diseases: an update for clinicians. Ann Nigerian Med 2010;4(2):37-44.

[6] Thomsen TW, Shaffer RW, Setnik GS. Nasogastric intubation. N Engl J Med 2006;354:e16.

[7] Intranasal analgesia prior to insertion of nasogastric tube- adults. Available at: http://prc.coh.org/FF\%20Intranasal-A3-11.pdf. 2017.

[8] Kuo YW, Yen M, Fetzer S, et al. Reducing the pain of nasogastric tube intubation with nebulized and atomized lidocaine: a systematic review and meta-analysis. JPSM 2010;40(4):613-20.

[9] Lemyze M. The placement of nasogastric tubes. CMAJ 2010;182(8):802.

[10] Metheny NA, Stewart BJ, Smith L, et al. pH and concentrations of pepsin and trypsin in feeding tube aspirates as predictors of tube placement. JPEN J Parenter Enteral Nutr 1997;21(5):279-85. 
[11] Metheny NA. Preventing respiratory complications of tube feedings: evidence-based practice. Am J Crit Care 2006;15(4):360-9.

[12] Ni M, Priest O, Phillips LD, et al. Risks of using bedside tests to verify nasogastric tube position in adult patients. EMJ Gastroenterol 2014;3:49-56.

[13] American Association of Critical-Care Nurses. AACN Practice Alert: Initial and ongoing verification of feeding tube placement in adults. Crit Care Nurse 2016;36(2):e8-e13.

[14] Giantsou E, Gunning KJ. Blindly inserted nasogastric feeding tubes and thoracic complications in intensive care. Health 2010;2(10):1135-41.

[15] Ozer S, Benumof JL. Oro- and nasogastric tube passage in intubated patients: fiberoptic description of where they go at the laryngeal level and how to make them enter the esophagus. Anesthesiology 1999;91(1):13743.

[16] Illias AM, Hui YL, Lin CC, et al. A comparison of nasogastric tube insertion techniques without using other instruments in anesthetized and intubated patients. Ann Saudi Med 2013;33(5):476-81.

[17] Flager N, Ball A. Easier nasogastric tube insertion. Anaesthesia 2004;59(2):197.

[18] Parris WC. Reverse sellick maneuver. Anesth Analg 1989;68(3):423.

[19] Appukutty J, Shroff PP. Nasogastric tube insertion using different techniques in anesthetized patients: a prospective, randomized study. Anesth Analg 2009;109(3):834-5.

[20] Tsai YF, Luo CF, Illias A, et al. Nasogastric tube insertion in anaesthetized and intubated patients: a new and reliable method. BMC Gastroenterol 2012;12:99.

[21] Chun DH, Kim NY, Shin YS, et al. A randomized, clinical trial of frozen versus standard nasogastric tube placement. World J Surg 2009;33(9):1789-92.

[22] Kirtania J, Ghose T, Garai D, et al. Esophageal guidewire-assisted nasogastric tube insertion in anesthetized and intubated patients: a prospective randomized controlled study. Anesth Analg 2012;114(2):343-8.

[23] Kumar P, Giridhar KK, Anand R, et al. Nasogastric tube placement in difficult cases: a novel and simple manoeuvre. J Anesth Clin Pharmacol 2005;21:429-34.

[24] Kayo R, Kajita I, Cho S, et al. A study on insertion of a nasogastric tube in intubated patients. Masui 2005;54(9):1034-6.

[25] Moharari RS, Fallah AH, Khajavi MR, et al. The glidescope facilitates nasogastric tube insertion: a randomized clinical trial. Anesth Analg 2010;110(1):115-8.

[26] Lai HY, Wang PK, Yang YL, et al. Facilitated insertion of a nasogastric tube in tracheal intubated patients using the glidescope. Br J Anaesth 2006;97(5):749-50.

[27] Hunter CW, Cohen S. A new use for the glidescope. Anesth Analg 2006;103(2):509.
[28] Boston AG. A novel endoscopic technique for failed nasogastric tube placement. Otolaryngology Head and Neck Surgery 2015;153(4):685-7.

[29] Okabe T, Goto G, Hori Y, et al. Gastric tube insertion under direct vision using the king vision ${ }^{\mathrm{TM}}$ video laryngoscope: a randomized, prospective, clinical trial. BMC Anesthesiol 2014;14:82.

[30] Doshi J, Anari S. Seldinger technique for insertion of a nasogastric tube. Laryngoscope 2006;116(4):672-3.

[31] Najafi M, Golzari SE. SORT maneuver for nasogastric tube insertion. Anaesthesia 2016;71(3):351.

[32] Gupta D, Agarwal A, Nath SS, et al. Inflation with air via a facepiece for facilitating insertion of a nasogastric tube: a prospective, randomized, double blind study. Anaesthesia 2007;62(2):127-30.

[33] Dobson AP. Nasogastric tube insertion-another technique. Anaesthesia 2006;61(11):1127.

[34] Ghatak T, Samanta S, Baronia AK. A new technique to insert nasogastric tube in an unconscious intubated patient. N Am J Med Sci 2013;5(1):68-70.

[35] Mandal MC, Dolai S, Ghosh S, et al. Comparison of four techniques of nasogastric tube insertion in anaesthetised, intubated patients: a randomized controlled trial. Indian J Anaesth 2014;58(6):714-8.

[36] Roubenoff R, Ravich WJ. Pneumothorax due to nasogastric feeding tubes. Report of four cases, review of the literature, and recommendations for prevention. Arch Intern Med 1989;149(1):184-8.

[37] Marderstein EL, Simmons RL, Ochoa JB. Patient safety: effect of institutional protocols on adverse events related to feeding tube placement in the critically ill. J Am Coll Surg 2004;199(1):39-47.

[38] Thomas BW, Falcone RE. Confirmation of nasogastric tube placement by colorimetric indicator detection of carbon dioxide: a preliminary report. J Am Coll Nutr 1998;17(2):195-7.

[39] Haddad N, Nawaz T, Potter L, et al. Avoidance of nasopulmonary intubation by feeding tubes with stethoscopic guidance the stethotube. Gastroenterology 1993;104(5):A623.

[40] Bercik P, Schlageter V, Mauro M, et al. Noninvasive verification of nasogastric tube placement using a magnet tracking system: a pilot study in healthy subjects. J Parenter Enteral Nutr 2005;29(4):305-10.

[41] Sorokin R, Gottlieb JE. Enhancing patient safety during feeding-tube insertion: a review of more than 2,000 insertions. J Parenter Enteral Nutr 2006;30(5):440-5.

[42] Metheny NA, Smith L, Stewart BJ. Development of a reliable and valid bedside test for bilirubin and its utility for improving prediction of feeding tube location. Nurs Res 2000;49(6):302-9.

[43] Araujo-Preza CE, Melhado ME, Gutierrez FJ, et al. Use of capnometry to verify feeding tube placement. Crit Care Med 2002;30(10):2255-9.

[44] Halloran O, Grecu B, Sinha A. Methods and complications of nasoenteral intubation. JPEN J Parenter Enteral Nutr 2011;35(1):61-6.

[45] Gupta PK, Gupta K, Jain M, et al. Postprocedural chest radiograph: impact on the management in critical care unit. Anesth Essays Res 2014;8(2):139-44. 